\section{A C G publications}

Bioorganic \& Medicinal Chemistry

Reports

\title{
Welcome to the first issue of BioorgMedChemRep for 2018!
}

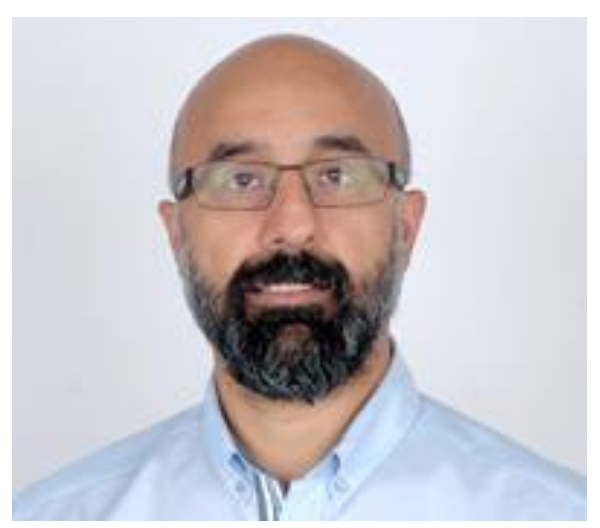

I am very excited to welcome you to the launch of BioorgMedChemRep-Bioorganic Medicinal Chemistry Reports a new, peer-reviewed, online journal that also supports open access. BioorgMedChemRep is ACG Publication's fourth journal that aimed to publish research related but not limited to the chemistry of medicine and its versatile applications. The aim of the journal is to promote a better understanding at the chemistry of the drugs and drug candidates at molecular level of life processes in the living organisms. The submissions are encouraged to contain medicinal chemistry and its applications. In addition to the original research articles, BioorgMedChemRep also accepts reviews, highlights and perspectives that would substantially contribute to the future studies in the bioorganic and medicinal chemistry research area.

The journal publishes research that addresses the biological questions at the molecular level, reports of experimental results in the chemical biology, medicinal chemistry and drug discovery as well as new and emerging advances and concepts in these fields.

My strong expectation is that this new journal will facilitate the publication process and will enable access to new research results, and foster the exchange of ideas among the multidisciplinary professionals and researchers involved in the medicinal chemistry field. With the help of a distinguished international editorial board of leading researchers around the world, BioorgMedChemRep aims to become one of the leading bridges that would close the gap between the researchers who are conducting studies with multi-disciplinary fashion, with the ultimate goal of improving the research in these fields.

On behalf of ACG Publications, I would like to take the opportunity to express our sincere gratitude to all authors, referees, Editorial Board Members, Editors and readers for their scientific and personal contributions to the BioorgMedChemRep.

It is a pleasure to announce the launching of the first issue of BioorgMedChemRep. I invite you to submit your work and become part of a growing community. We look forward to working with you to improve the standards and products of the bioorganic and medicinal chemistry.

Oztekin Algul, $\mathrm{PhD}$

Editor-in-Chief of BioorgMedChemRep Professor at the Pharmaceutical Chemistry Department, Mersin University Mersin Turkey E-mail address: oztekin.algul@ acgpubs.org 